\title{
The Impact of the COVID-19 Pandemic on Students' Body Posture During Online Learning Should not be Overlooked
}

\author{
Sajad Bagherian (iD) ${ }^{1, *}$ and Simin Nafian (iD ${ }^{2}$ \\ ${ }^{1}$ Department of Sports Sciences, Shahrekord University, Shahrekord, Iran \\ ${ }^{2}$ Department of Stem Cell and Regenerative Medicine, Institute of Medical Biotechnology, National Institute of Genetic Engineering \& Biotechnology (NIGEB), Tehran, Iran \\ "Corresponding author: Department of Sports Sciences, Shahrekord University, Shahrekord, Iran. Email: s.bagherian@sku.ac.ir
}

Received 2021 July 25; Revised 2021 September 22; Accepted 2021 September 24.

Keywords: COVID-19, Health, Home

\section{Dear Editor,}

The lives of millions of people across the world have changed following the coronavirus disease 2019 (COVID19) pandemic (1). According to the United Nations Educational, Scientific and Cultural Organization (UNESCO), the COVID-19 pandemic has resulted in the closure of schools and the disruption of education for more than $60 \%$ of the world's students (1). In many nations throughout the world, schools are still closed as part of the COVID-19 pandemic response (2). Governments are dealing with a growing number of questions concerning when and how to reopen schools (2). Different countries worldwide have introduced various solutions, such as generating various online platforms during the pandemic to continue the education process (3).

In the current educational systems, children are required to sit for long periods of time, and the increased use of classroom-based technology adds to the number of hours spent sitting (4). Long periods of sitting have been linked to the development of back discomfort (5). Students who participate in distance/online learning may also use different habitual postures (e.g., sitting in a slouched posture, lying supine or in a prone position, sitting at the edge of the chair, etc.) during their virtual learning experiences (4). These postures represent poor body alignment positions that cause micro spasms, a decrease in normal elasticity of the soft tissue, and changes in the length and strength of muscles that alter the relative participation of the antagonists and synergists. The type of sustained or awkward posture that students perform during the day is probably a major contributory factor to functional postural disorders (e.g., forward-head, kyphosis, lordosis, sway- back, etc.) (6). Prolonged postures during online learning may result in tissue adaptations (i.e., short, stiff, long, overused, or weak muscles). When muscle length is altered due to misalignment (i.e., poor posture), tension development will be decreased, and the muscle is unable to generate the necessary force for efficient posture (7). For example, a postural disorder caused by improper sitting, can result in increased neck, mid-, and lower back, shoulder, and leg pain (7).

For all stakeholders, including students, teachers, parents, researchers, and physicians, knowing whether postural habits are a source of postural disorders is critical (8). Early detection and treatment of functional postural disorders may help to reduce their complications. Individuals with postural disorders may benefit from corrective exercises to improve muscle imbalance, strength, and flexibility. COVID-19 has the potential to exacerbate physical inactivity and reduce access to organized sports, both of which have long been identified as public health concerns (9). It appears that home-based corrective exercise programs can represent a new approach to improve functional postural disorders during the COVID-19 pandemic (Figure 1). Regular home-based activities may be beneficial in keeping students active, preventing some physical inactivity issues, and helping individuals to stay fit and healthy (10). To avoid the airborne coronavirus and maintain fitness levels, a home-based exercise program using several safe, easy, and simply implementable exercises is recommended. This program can include aerobic (house walking), strengthening, stretching, and balance activities, or a combination of all (11). Due to the health advantages of regular physical activity, the World Health Organization 
(WHO) recommends that people aged 5-17 years engage in at least 60 minutes of moderate- to vigorous-intensity physical activity every day throughout the week (12). In addition, vigorous-intensity activities, including aerobic and activities that strengthen bone and muscle, should be included at least three days a week (12).

In conclusion, students' families and health and fitness providers must educate these young populations about postural disorders. Therefore, students aware of the consequences of poor posture during online learning must try and use the recommended and safe postures. Conversely, it is usually essential to enrich and broaden the use of corrective exercises for improving postural patterns, both therapeutically and in routine practice. Hence, during the COVID-19 pandemic, using home-based corrective exercises to minimize functional postural disorders is recommended. Future research should investigate the beneficial health effects of various types of exercise that can be used to alleviate bad posture. The selection of exercises should include routines that can be performed at home safely by children and adolescents.

\section{Footnotes}

Authors' Contribution: Sajad Bagherian and Simin Nafian provided materials and edited the documents and Sajad Bagherian was the lead author.

Conflict of Interests: The authors declared that they have no known competing interests.

Ethical Approval: Not applicable for this manuscript Funding/Support: None declared.

\section{References}

1. Edmunds WJ. Finding a path to reopen schools during the COVID19 pandemic. Lancet Child Adolesc Health. 2020;4(11):796-7. doi: 10.1016/S2352-4642(20)30249-2. [PubMed: 32758452]. [PubMed Central: PMC7398667].

2. Viner RM, Bonell C, Drake L, Jourdan D, Davies N, Baltag V, et al. Reopening schools during the COVID-19 pandemic: governments must balance the uncertainty and risks of reopening schools against the clear harms associated with prolonged closure. Arch Dis Child. 2021;106(2):111-3. doi: 10.1136/archdischild-2020-319963. [PubMed: 32747375]. [PubMed Central: PMC7401577].

3. Adnan M. Online learning amid the COVID-19 pandemic: Students perspectives. J Pedagog Sociol and Psychol. 2020;1(2):45-51. doi: 10.33902/jpsp.2020261309.

4. Bettany-Saltikov J, McSherry R, Schaik P, Kandasamy G, Hogg J, Whittaker V, et al. PROTOCOL: School-based education programmes for improving knowledge of back health, ergonomics and postural behaviour of school children aged 4-18: A systematic review. Campbell Systematic Reviews. 2019;15(1-2). doi:10.1002/cl2.1014.

5. Lis AM, Black KM, Korn H, Nordin M. Association between sitting and occupational LBP. Eur Spine J. 2007;16(2):283-98. doi: 10.1007/s00586006-0143-7. [PubMed:16736200]. [PubMed Central: PMC2200681].

6. Solberg G. Postural disorders and musculoskeletal dysfunction: diagnosis, prevention, and treatment. Elsevier Health Sciences; 2007.

7. Clark M, Lucett S. NASM essentials of corrective exercise training. Lippincott Williams \& Wilkins; 2010.

8. Dugan JE. Teaching the body: A systematic review of posture in terventions in primary schools. Educ Rev. 2017;70(5):643-61. doi: 10.1080/00131911.2017.1359821.

9. Fitzpatrick J, Castricum A, Seward H, Tulloh L, Dawson E. Infographic. COFIT-19: let's get moving through the COVID-19 pandemic!. BrJSports Med. 2020;54(22):1360-1. doi: 10.1136/bjsports-2020-102661. [PubMed 32561519].

10. Halabchi F, Ahmadinejad Z, Selk-Ghaffari M. COVID-19 epidemic: Exercise or not to exercise; that is the question!. Asian J Sports Med. 2020;11(1). e102630. doi: 10.5812/asjsm.102630.

11. Chen P, Mao L, Nassis GP, Harmer P, Ainsworth BE, Li F. Coronavirus disease (COVID-19): The need to maintain regular physical activity while taking precautions. J Sport Health Sci. 2020;9(2):103-4 doi: 10.1016/j.jshs.2020.02.001. [PubMed: 32099716]. [PubMed Central: PMC7031771].

12. WHO. WHO guidelines on physical activity and sedentary behaviour: at a glance. 2020 


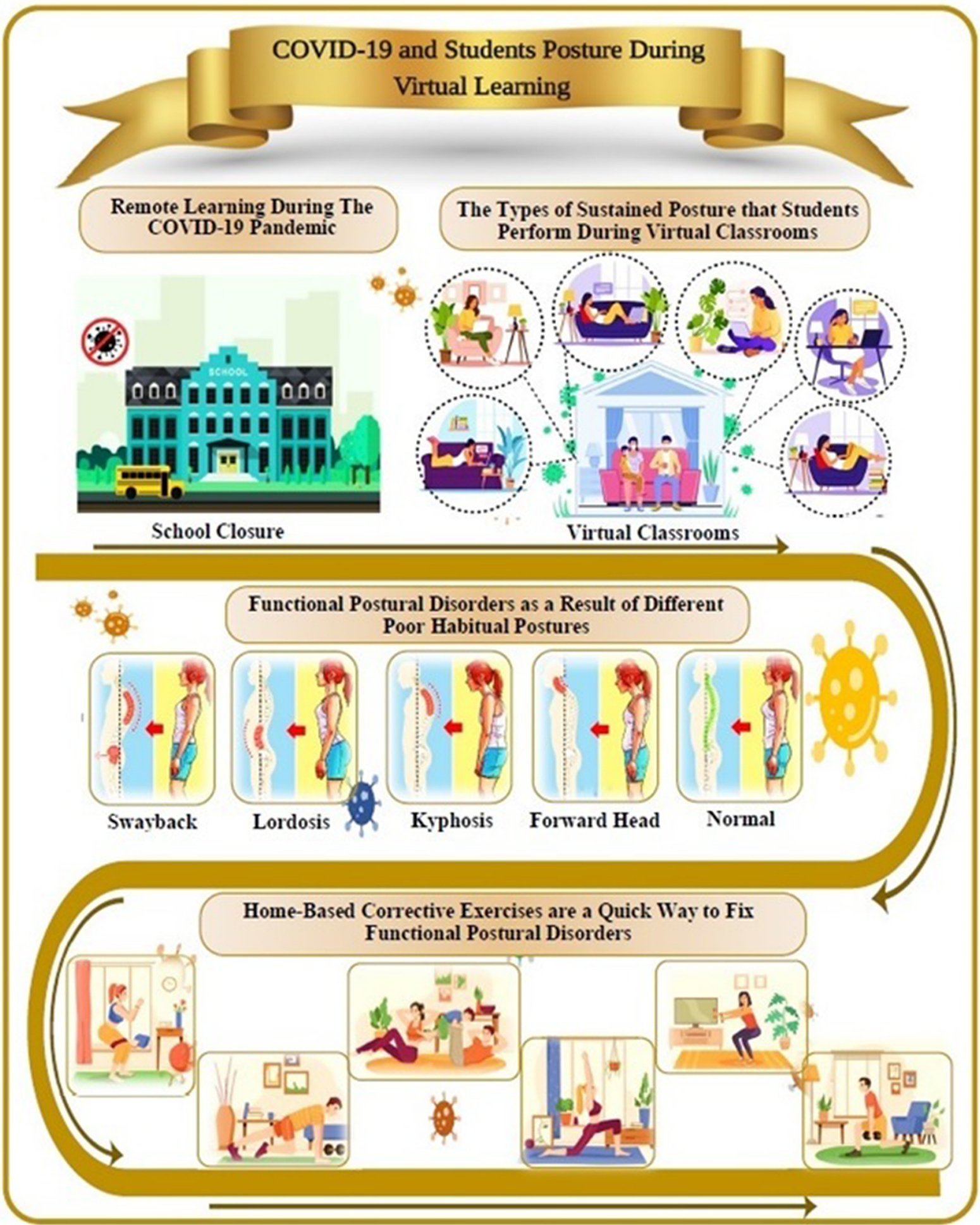

Figure 1. Interrelationships between school closure and students' body posture. 